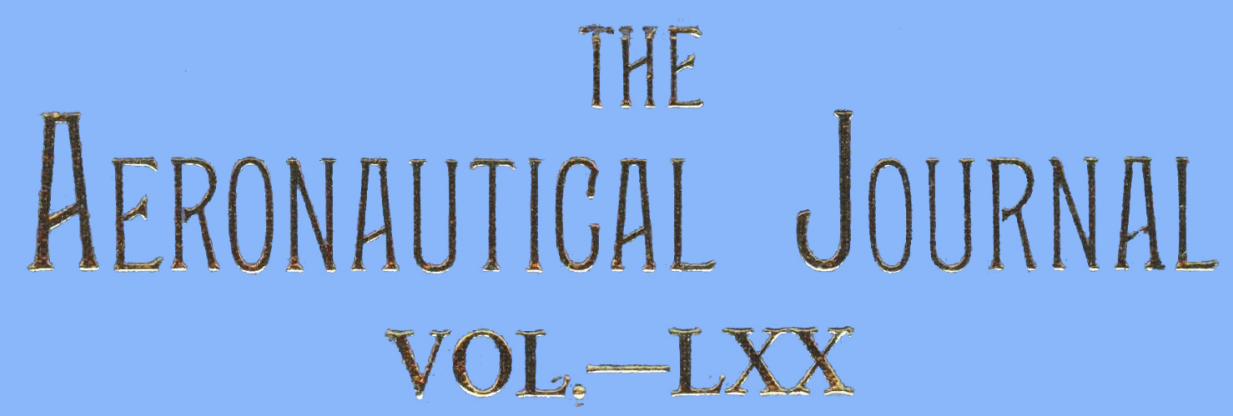

THE ROYAL AERONAUTICAL SOCIETY 


\section{The Journal of the Royal Aeronautical Society}

INCORPORATING THE INSTITUTION OF AERONAUTICAL ENGINEERS AND THE HELICOPTER ASSOCIATION OF GREAT BRITAIN

\section{VOLUME LXX}

JANUARY - DECEMBER 1966

Edited for the Council of The Royal Aeronautical Society

by

Joan Bradbrooke, ARAeS 


\section{Journal of \\ THE ROYAL AERONAUTICAL SOCIETY}

INCORPORATING THE INSTITUTION OF AERONAUTICAL ENGINEERS AND THE HELICOPTER ASSOCIATION OF GREAT BRITAIN

Published Monthly at 4 HAMILTON PLACE, LONDON W1

Telephone: Grosvenor 3515-9 Telegrams: Didaskalos, London, W1

Subscriptions: 610 10s. Od. per annum, post free Single Copies: 17s. 6d.

Advertisements only: J. W. Williams, DFC, Magazine Advertising Ltd, 36-38 Whitefriars St., London, EC4. Fleet Street 7539

\begin{tabular}{llll}
\hline \hline VOLUME 70 & SEPTEMBER 1966 & NUMBER 669 \\
\hline
\end{tabular}

\section{CONTENTS}

Page

Notices

LXV

New Fellows-Royal Flying Corps Memorial-Courses on Air Law-Graduates' and Students' Section Diary-Branches' Centenary Celebrations: September and October-The Council of Engineering Institutions News-Acknowledgments-Library Note-Diary-Elections-Other Meetings-News of Members-Lecture Summaries.

STOL-Some Possibilities and Limitations

W. A. Mair

The Society of British Aerospace Companies Ltd

H. W. Goodinge

836

The UK Polaris Project

Captain C. W. H. Shepherd

843

Economic Space Transportation-Thoughts on Missions, Size and Operational Sensitivity

T. Derbyshire, W. B. Clegg and T. W. Smith

Propulsion Controls on the Concorde

P. Young

Correspondence

Technical Notes

A Generalised System for Project Control and Evaluation-G. Mitchell. A Short Proof of the PrandtlMeyer Expansion Formula-A. R. Collar. The Steady Flow Through a Cascade of Closely Spaced Circular Cylinders-B. W. Roberts.

The Library

Reviews: Roots of British Air Policy-The Invention of the Aeroplane, 1799-1909-The Matrix Analysis of Vibration.

Additions to the Library

Reports

\begin{abstract}
All communications for publication in the JOURNAL should be addressed to the Editor, JOAN BRADBROOKE, MBE, ARAeS; all communications on general matters affecting the Society should be addressed to the Secretary, A. M. BALLANTYNE, OBE, TD, BSC, PhD, CEng, FRAeS, HonFCASI, FAIAA - ot 4 HAMILTON PLACE, LONDON, W1.
\end{abstract}

Reproduction in whole or in part of any of the papers and contributions published in this Journal is not permitted without the written consent of the Royal Aeronautical Society.

None of the papers or paragraphs must be taken as expressing the opinion of the Council of the Royal Aeronautical Society unless otherwise stated. 


\section{THE ROYAL AERONAUTICAL SOCIETY}

\section{OVERSEAS DIVISIONS}

\section{Australia}

President: D. B. HUDSON, FRAeS.

Hon. Secretary: W. ISBISTER, FRAeS, Science House, Gloucester \& Essex

Branches at: Canberra, Sydney, Melbourne and Adelaide.

\section{Rhodesia}

Hon. President: Sir HUMPHREY GIBBS, KCMG, OBE.

Hon. Secretary: S. H. GUY, AFRAeS, c/o Central African Airways, Private Bag 1 ,

\section{New Zealand}

President: Gp. Capt. W. G. WOODWARD OBE, AFRAeS.

Hon. Secretory: T. T. N. COLERIDGE, AFRAeS, The Shell Company of New Zealand Ltd, Box 2091, Wellington.

Branches at: Auckland, Wellington, Palmerston North and Christchurch.

\section{Southern Africa}

President: Dr. C. G. van NIEKERK, AfRAeS.

Hon. Secretory: A. W. BENNELL, PO Box 4409, Johannesburg.

Branches at: Johannesburg, Pretoria and Cape Town.

\section{BRANCHES IN THE UNITED KINGDOM}

\section{Bedford}

President: J. HANSON, FRAeS.

Chairman: F. O'HARA, AFRAeS.

Hon. Secretary: T. E. B. BATEMAN, AFRAeS, Aircraft Research Association, Manton Lane, Bedford. Tel: Bedford 67701 .

\section{Belfost}

President: C. E. WRANGHAM.

Chairman: E. G. COLLINSON, AFRAeS.

Hon. Secrezary: D. SAUNDERS, AFRAeS, Short Bros \& Harland Ltd, Castlereagh, Belfast.

Birmingham and Wolverhampton

President: H. S. BOTTOMS, AFRAeS.

Chairman: N. H. SHUTTLEWORTH, AFRAES.

Hon. Secretary: G. O. FRAMPTON, AFRAeS, H.M. Hobson Ltd, Fordhouses, Wolver hampton, Staffs. Tel: Fordhouses 2381 .

\section{Boscombe Down}

President: Air Cdre. F. R. BIRD, DSO, DFC, AFC. Chairman: G. MACLAREN-HUMPHREYS, MBE, AFRAeS.

Hon. Secretary: San. Ldr. H. H. HORSHAM, MBE, ARAeS, A \& AEE, Boscombe Down, Amesbury,

Bristol

President: Dr. R. R. JAMISON, FRAeS.

Chairman: G. L. AUTY, FRAeS.

Hon. Secretary: W. O. LEE, ARAeS, BAC (Op) Ltd, Filton Division,
693831, Ext 449.

\section{Brough}

President: Capt. E. D. G. LEWIN, CB, CBE, DSO, AFRAeS.

Choirman: R. D. BOOT, AfRAeS.

Hon. Secretary: A. D. HOWARTH, ARAeS, c\% Hawker Siddeley Aviation Ltd, Brough. Yorkshire. Tel: Brough 667121, Ext 314.

\section{Cambridge}

President: Profossor W. A. MAIR, FRAoS.

Choirman: R. O. GATES, AFRAeS.

Hon. Secretary: N. A. J. HARRY, AFRAeS, Marshall of Cambridge (Engineering) Ltd, Aircraft Division, Airpo

\section{Chester}

President: C. B. V. NEILSON, FRAeS.

Chairman: F. G. EVANS, FRAES

Hon. Secretary: H. PENDLEBURY, AFRAES, ElectroHydraulics Ltd, Liverpool Road, Warrington, Lancs.

\section{Christchurch}

President: E. W. J. GRAY, FRAeS. Chairman: H. E. VINCENT, AFRAES.

Hon. Secretary: J. F. BRENNAN, AFRAeS, c\% 'BAC (Op) Ltd, Weybridge Division, BourneBAC (Op) Ltd, Weybridge Division, Bournemouth (Hurn) Airport.

\section{Coventry}

President: J. WRIGHT, CBE, FRAeS.

Chairmon: D. A. WOODLEY, FRAES.

Hon. Secretary: G. W. COLLINS, AFRAeS, Rocket Dept, Bristol Siddeley Engines Ltd. Ansty Aerodrome, P.O. Box 17, Coventry Warwickshire. Tel: Walsgrave-on-Sowe 3211.

\section{Cranwell}

President: Air Vice-Marshal I. D. N. LAWSON, CB, CBE, DFC

Chairman: Wg. Cdr. J. SANDFORD, AFRAeS.

Hon. Secretary: F. A. BRISTOW, AFRAES, Dept of Engineering, RAF College, Cranwell, Sleaford, Lincs. Tel: Cranwell 201.

\section{Derby}

President: Sir DENNING PEARSON, HonfRAeS. Chairman: H. PEARSON, FRAES.

Hon. Secretary: V. R. HAAS, AFRAeS, Rolls-Royce Ltd, Derby, Tel: Derby 42424.

\section{Glasgow}

President: Dr. A. W. BABISTER, AFRAeS.

Chairman: Wm. H. G. FINLAY, AFRAeS.

Hon. Secretary: W. N. ALCOCK, Shenstone, Dumbuck Crescent, Dumbarton.

\section{Gloucester and Cheltenham}

President: Sir GEORGE DOWTY, FRAeS

Chairman: J. G. RUSSELL, AFRAeS.

Hon. Secretary: C. E. HUTT, ARAeS, c/o Smiths Industries Ltd, Bishops Cleeve, Cheltenham Glos. Tel: Bishops Cleeve 3333 .

\section{Halton}

President: Air Vice-Marshal B. ROBINSON, CBE. Chairman: Gp. Capt. V. C. G. USHER, AFRAeS. Hon. Secretary: San. Ldr. J. MACKINTOSH, Officers' Mess, RAF Halton, Bucks. Tel: Wendover 2242, Ext 310 .

\section{Hotfield}

President: Air Cdre.F. R. BANKS, CB, OBE, FRAeS. Chairman: E. B. ROBINSON, AFRAeS.

Hon. Secretary: G. B. EVANS, AFRAeS, Drawing Office, Hawker Siddeley Aviation Lto

\section{Isle of Wight}

President: H. KNOWLER, FRAeS.

Chairman: G. J. THOMPSON, FRAeS.

Hon. Secretary: B. J. SUMMERS, GradRAeS, Dosign Division, Osborne, Isle of Wight. Tel: Cowes 4121.

\section{London Airport}

President: Rear Admiral Sir MATTHEW S. SLATTERY, KBE, CB, FRAES.

Chairman: J. R. FINNIMORE, AFRAeS.

Hon. Secretary: N. BROWN, ARAeS, Technical Publications, Technical Block A, BOAC, London (Heathrow) Airport. Hounslow, Middlesex. Tel: Skyport 5511, Ext 2883.

\section{Loughborough}

President: Professor K. L. C. LEGG, FRAeS.

Chairman: K. B. AYERS, AFRAES.

Hon. Secretary: F. G. MACCABEE, AFRAeS, 56 Holywell Drive, Loughborough, Leics.

\section{Luton and Stevenage}

President: K. D. MORGAN.

Chairman: H. E. CURTIS, AFRAES,

Hon. Secretary: P. EVERARD, AFRAeS, c/o BAC (Op) Ltd, Guided Weapons Div., Technical Design Dept., Six Hills Way, Stevenage, Herts. Tel: Stevenage 2422.

\section{Manchester}

President: C. E. FIELDING, OBE, FRAES. Chairman: M. J. BRENNAN, FRAeS.

Hon. Secretary: J. A. E. WATERfALL, 56 Manor Avenue, Ashton-on-Mersey, Cheshire. Tel: Failsworth 2020

\section{Merthyr and Treforest}

President: E. J. BUSH.

Chairman: E. R. CHAYTOR, AFRAeS.

Hon. Secretary: Dr. G. O. FORESTER, Chief of Research, Teddington Aircrait Controls Led Merthyr Tydfil 3261 .

\section{Preston}

President: F. W. PAGE, CBE, FRAeS.

Chairman: E. LOVELESS, AFRAES.

Hon. Secretary: K. HOLTAPPEL, Design Office, BAC (Op) Ltd, Preston Division, Warton Aerodrome, Preston, Lancashire. Tel: Freckleton 531.

\section{Prestwick}

President: R. McINTYRE, FRAeS.

Chairman: T. C. OVEREND, ARAeS

Hon. Secretary: J. LOGAN, AFRAeS, Scottish Aviation Ltd, Service, Dept, Prestwick

\section{Reading}

President: E. N. MOBBS.

Chairman: G. BOYCE, AFRAeS.

Hon. Secretary: E. W. C. ANGELL, ARAeS, M. L Aviation Co Ltd, White Waltham, Maidenhead. Berks. Tel: Littlowick Green 248 .

\section{Southampton}

President: Professor E. J. RICHARDS, OBE, FRAoS. Chairman: F. C. KIRKPATRICK, AFRAeS.

Hon. Secretary: P. W. FORTESCUE, AFRAeS, Dept. of Aeronautics and Astronautics, The Unive rsity,

Southend

President: 1. R. BATT, ARAeS.

Chairman: K. A. COLE, AFRAeS.

Hon. Secretary: Wg. Cdr. E. F. PRICE, ARAeS, Aviation Traders (Eng) Led, Southend Airport, Aviation Traders (Eng) Ltd,

\section{Swindon}

President: Gp. Capt. F. S. HAZLEWOOD, OBE,

Chairman: W. GILLESPIE, AFRAoS.

Hon. Secretary: E. J. DAVIS, AFRAoS, Viekers Ltd, South Marston, Swindon, Wilts. Tel: Stratton St. Margaret 3241.

\section{Weybridge}

President: Sir GEORGE EDWARDS, CBE, HonfRAeS.

Chairman: J. HEAP, AFRAeS.

Hon. Secretary: A. M. DOBSON, AFRAES, BAC (OP) Ltd, Weybridge Division, Weybridge. Surrey. Tel: Weybridge 45555.

Yeovil

President: E. C. WHEELDON, CBE, FRAeS.

Chairmen: Dr. G. S. HISLOP, FRAeS and Dr. E. W. STILL, FRAES.

Hon. Secretary: L. A. LANSDOWN, AFRAeS, Tel: Yeovil 5222 